\title{
Investigating polystyrene nanoplastics-induced reproductive toxicity in vitro: Focus on Nrf2-PKM2-autophagy signaling pathway
}

\section{Siwen Li ( $D$ lisiwen0529@163.com)}

Central South University https://orcid.org/0000-0003-0784-134X

\section{Yu Ma}

Central South University

Shuzi Ye

Central South University

Ying Su

Central South University

Die Hu

Central South University

Fang Xiao

Central South University

\section{Research Article}

Keywords: Nanoplastics, Mitochondrial apoptosis, Nuclear factor erythroid-derived 2-related factor (Nrf2), Pyruvate kinase M2, Reproductive toxicity

Posted Date: October 19th, 2021

DOI: https://doi.org/10.21203/rs.3.rs-872932/v1

License: (1) (1) This work is licensed under a Creative Commons Attribution 4.0 International License. Read Full License 


\section{Abstract}

As an issue of widespread concern, microplastics pollution has emerged as a harmful environmental pollutant. Nanoplastics (NaPs) has reported to accumulate in the testes and cause degeneration in the seminiferous tubules. However, the current research involving NaPs-induced reproductive toxicity remains poorly understood. The current work aimed to investigate the mechanisms of NaPs-induced reproductive injury in vitro. At first, we found that $80 \mathrm{~nm}$ fluorescent NaPs could enter into GC$2 s p d(t s)$ cells by fluorescent inverted microscope. Our results also demonstrated that suppression of reactive oxygen species (ROS) inhibited NaPs-triggered mitochondrial apoptosis and autophagy in GC-2spd(ts) cells. We also found that NaPs treatment did not change the interaction between nuclear factor erythroid-derived 2-related factor (Nrf2) and Kelch-like ECH associated protein 1 (Keap1), while inhibiting nuclear accumulation of Nrf2 protein. Further in vitro experiments showed that NaPs-induced reproductive toxicity associated with reducing dimerize pyruvate kinase M2 (PKM2), which are ascribed to the loss of Nrf2. Meanwhile, improving nuclear accumulation of Nrf2 might interact with PKM2 to rescue mitochondrial apoptosis caused by NaPs. Together, this study highlight that disturbing Nrf2-PKM2 signaling is essential process of NaPs-induced reproductive toxicity and provide valuable insights into the mechanism of microplastics-induced reproductive toxicity.

\section{Introduction}

Plastic has become an integral part of modern world, penetrating basically every aspect of our lives. It has been estimated that worldwide plastic production reached 550 million tonnes per year in 2018 (Plastics Europe, 2018). However, a staggering 32\% of plastic packaging escapes collection systems owing to low public awareness and mismanagement of plastic waste. It was estimated that poor recycling use has contributed to the accumulation of 15-51 trillion of plastic particles floating on the surface of the oceans (Van Sebille et al., 2015). If improper human behavior and waste mismanagement trends continue, approximately 12,000 tons of plastic waste will eventually enter the natural environment by 2050 (Geyer et al., 2017). Large plastics gradually undergo weathering processes, ultraviolet radiation and microbial breakdown to form the microplastics (particles $<5 \mathrm{~mm}$ ). Emerging evidence suggests that microplastics are easily transferred and accumulated along the food chain, and eventually enter the human body, posing potential threats to human health (Rahman et al., 2021). Hence, microplastics are recognized as an ecological risk to human health in recent years.

Microplastics are absorbed from the gastrointestinal tract lumen via the endocytosis of $\mathrm{M}$ cells, and subsequently transported to the mucosal lymphoid tissues, thereby entering circulatory system (Wright and Kelly, 2017). In recent mammalian experiments demonstrated that microplastics could induce hepatotoxicity, cardiovascular toxicity and behavioral disorders via ingestion or inhalation does occur (Deng et al., 2017; Wright and Kelly, 2017). In addition, rats showed cardiomyocyte apoptosis after being given high doses of microplastics (50 mg/L) for 90 consecutive days, which may be correlated with burst generation of reactive oxygen species (ROS) (Li et al., 2020). It is noted that the reproductive system is one of the most sensitive systems in an organism in microplastics exposure (Yin et al., 2021). Further study reported that polystyrene microplastics accumulates in the testis of mice, causing blood-testis barrier disruption and sperm DNA damage (Jin et al., 2021; Hou et al., 2021a). Balb/c mice exposed to 1 $\mathrm{mg}$ /day of polystyrene microplastics showed deterioration of sperm parameters through enhancing ROS production (Deng et al., 2021; Jin et al., 2021; Hou et al., 2021). Coincidentally, male rats treated with $10 \mathrm{mg} / \mathrm{kg} /$ day polystyrene nanoparticles (NaPs) showed signs of testicular atrophy and seminiferous tubule degeneration (Amereh et al., 2020). On the other hand, testis accumulation of NaPs could cause alterations in sperm physiology and spermatogenesis disorders (Deng et al., 2021). However, the present knowledge on reproductive toxicity posed by nanoparticles is still incomplete.

Previous study suggest that Excessive ROS is often a hallmark feature in damaged spermatogenesis, which in turn implies its role in the development of impaired reproductive function (Sedha et al., 2015). Uncontrolled ROS production is a driving force for mitochondrial damage in fertility impairment, while nuclear factor erythroid-derived 2-related factor (Nrf2) is responsive to the alternations in cellular redox homeostasis. We have reported that Nrf2 signaling protects adverse effects of microplastics in vivo and in vitro (Li et al., 2021). As a next step in ROS burst event, Nrf2 dissociates from Keap1 and enters the nucleus to transactivates target gene, such as heme oxygenase-1 (HO-1) and NAD (P)H dehydrogenase quinone 1 (NQ01). Despite the fact that Nrf2 activation is a protective regulator against testicular injury, burgeoning studies have shown the deleterious side of Nrf2 (Zhang et al., 2019; Zhang and Chapman, 2020). Also, it is confirmed that Nrf2 down-regulation is involved in prepubertal testis 
injury (Zhao et al., 2020). Hence, maintaining the redox balance is important to mitigate microplastics-induced reproductive toxicity.

Pyruvate kinase M2 (PKM2) is a critical rate-limiting enzyme of aerobic glycolysis and catalyzes the reaction of phosphoenolpyruvate to pyruvate with the generation of ATP. In addition to its metabolic functions (tetramer PKM2), dimeric PKM2 exhibits kinase potential beyond glycolysis involved in signal transducer and activator of Nrf2 (Luo et al., 2011; Wei et al., 2020). Previous evidence suggested that nuclear PKM2 promotes transcription of Hif-1a by phosphorylating STAT3 at tyrosine 705 (Dong et al., 2015). Of note, recent research implied that pharmacologic PKM2 inactivation blocked the abnormal cell cycle and apoptosis in vitro (Zheng et al., 2020). Additionally, abnormal expression of PKM2 was observed in many pathological states, including formaldehyde-induced neurotoxicity and arsenic-triggered hepatotoxicity (Wang et al., 2020a; Li et al., 2021). Therefore, we hypothesized that PKM2/Nrf2 signal regulatory oxidative stress and metabolic functions interaction mechanism is involved in microplastics-triggered reproductive toxicity.

Environmental contaminants-induced reproductive toxicity is an area of great concern. Mice spermatocyte cells (GC-2spd cells) have been used in many studies as an in vitro model for reproductive toxicological research (Ren et al., 2019; Jin et al., 2021). According to above studies, we hypothesized that Nrf2-mediated anti-oxidant defence should be an essential target for the molecular mechanism of NaPs-triggered reproductive toxicity. On the other hand, we also investigated the roles of Nrf2-PKM2 signaling in NaPs-induced reproductive toxicity in the GC-2spd cells.

\section{Materials And Methods}

\subsection{Chemicals and Reagents}

NaPs with a particle size of $80 \mathrm{~nm}$ was obtained from TianJin Baseline Chromtech Research Center (Tianjin, China). The fluorescence microcopy images of NaPS was shown in the Fig. 1A. According to scanning electron microscope analysis, the NaPs used in this study were spheres (Fig. 1B). Figure 1C showed the absorption peak when the maximum excitation and emission wavelengths at $488 \mathrm{~nm}$ and $518 \mathrm{~nm}$, respectively. Additionally, the properties of nanoplastics used in this study was shown in Table 1. ML385 (HY-100523) were purchased from Med Chem Express (Brea, CA, USA). mTORC (A11354) and FITC-conjugated goat anti-mouse $\operatorname{lgG}(\mathrm{H}+\mathrm{L})$ (AS001) were obtained from ABclonal Technology Co.,Ltd. (Wuhan, China). Nrf2 (16396-1-AP), Keap1 (10503-2-AP), Nqo1 (67240-1-lg), Ho-1 (10701-1-AP), p62 (18420-1-AP), GLUT1 (glucose transporter, 21829-1-AP), HKII (hexokinase 2, 22029-1-AP), PKM2 (15822-1-AP) and Cy3-conjugated goat anti-rabbit IgG(H+L) (SA00009-2) were obtained from proteintech (Wuhan, China). Antibodies against LC3B-I/II (4108S) was obtained from Cell Signaling Technology (Beverley, MA). Sodium hydrosulfide hydrate (cas: 207683-19-0) was obtained from Shanghai Rhawn Chemical Technology Co., Ltd. 4',6diamidino-2-phenylindole (DAPI), ROS Assay kit and JC-1 Mitochondrial Membrane Potential Assay Kit were purchased from Beyotime Life Science Inc. (Shanghai, China). Pierce Co-immunprecipitation Kit (26149) was obtained from Thermo Scientific. $5 \times$ Native Sample Loading Buffer (C506032) was obtained from Sangon Biotech Co., Ltd. (Shanghai, China).

\subsection{Cell culture and stimulation}

Mouse spermatocyte-derived GC-2spd(ts) cells were purchased from Procell Life Science\&Technology Co.,Ltd.. GC-2spd(ts) cells were grown in DMEM supplemented with $12 \% \mathrm{FBS}$ and $1 \%$ penicillin/streptomycin in $5 \% \mathrm{CO}_{2}$ at $37{ }^{\circ} \mathrm{C}$.

GC-2spd(ts) cells were incubated for $24 \mathrm{~h}$ with NaPs $(400 \mu \mathrm{g} / \mathrm{mL})$. The concentration of NaPs was chosen to assess the microplastic reproductive toxicity based on previous work (Hou et al., 2021). To determine the beneficial effects on NaPs-triggered reproductive toxicity in vitro, cells were stimulated with NaHS $(250 \mu \mathrm{M}) 1 \mathrm{~h}$ before NaPs treatment. Next, to confirm the roles of $\mathrm{Nrf2}$ in endogenous $\mathrm{H}_{2} \mathrm{~S}$-triggered protective effects, we pre-treated GC-2spd(ts) cell with Nrf2 inhibitor ML385 and NaHS for $1 \mathrm{~h}$ and cultured with NaPs for for another $24 \mathrm{~h}$ for further analysis.

\subsection{Cell-apoptosis analysis}

After treatment with reagent for the indicated duration, the percentage of apoptotic cells were determined by Annexin V-PE/7-AAD Detection Kit (Procell, Wuhan, China) as described (Li et al., 2021). A total of $1 \times 10^{6}$ cells were collected and washed twice with 
PBS. Then, the cells were resuspended with $500 \mu \mathrm{L}$ binding buffers containing $5 \mu \mathrm{L}$ 7-AAD and $5 \mu \mathrm{L}$ Annexin V-PE. Following a 20 min incubating in the dark at $37^{\circ} \mathrm{C}$, Next, the cells were read in the flow cytometer Beckman CytoFLEX FCM. FlowJo software was utilized to calculate the percentage of apoptotic cells.

\subsection{Assessing mitochondrial membrane potential (MMP)}

After treatment with reagent, the cells were incubated with $1 \times \mathrm{JC}-1$ dye for $30 \mathrm{~min}$ at $37^{\circ} \mathrm{C}$. Then, the cells were harvested and washed with JC-1 staining buffer. Next, the ratios of red/green fluorescence levels was recorded by flow cytometry. Additionally, cells were observed by inverted microscope (EVOS ${ }^{\text {Tw }}$ M7000 Imaging System, Thermo Scientific).

\subsection{Detection of endogenous ROS levels}

The concentration of ROS was measured using the DCFH-DA probe. After pertreating with NaHS or/and ML385 followed by NaPs stimulation, $10 \mu \mathrm{M}$ DCFH-DA was added to the cells culture medium at $37^{\circ} \mathrm{C}$ for $30 \mathrm{~min}$. Next, the cells were washed with serumfree medium. The levels of ROS were recorded using flow cytometry (Beckman CytoFLEX FCM). The fluorescence levels was quantified using FlowJo software.

\subsection{Western blotting analysis}

Cells were harvested and lysed with RIPA buffer with 1 mM PMSF (Beyotime Biotechnology, Shanghai, China). $25 \mu \mathrm{g}$ of protein was separated with $12 \%$ SDS-PAGE and then electroblotted onto PVDF membranes. Next, the membranes were blocked with $5 \%$ nonfat milk in TBST for $1 \mathrm{hr}$ at $37^{\circ} \mathrm{C}$. Thereafter, the membranes were probed overnight at $4^{\circ} \mathrm{C}$ with primary antibodies. Antibodies used and the diluted ratio were shown in Supplementary Materials. After three washes with TBST, the blots were incubated with the related secondary antibodies for $1 \mathrm{hr}$ at $37^{\circ} \mathrm{C}$. The images of target proteins were visualized using Azure Biosystems (USA). The bands were quantified by the Image $\mathrm{J}$ software and normalized to $\beta$-actin.

\subsection{Immunofluorescent staining}

After fixed with $4 \%$ paraformaldehyde, the GC-2spd(ts) cells were permeabilized with $0.5 \%$ Triton X-100/PBS for 20 min. The samples were blocked with $5 \%$ BSA before being incubated with Nrf2, PKM2, LC3-II in a wet box at $4^{\circ} \mathrm{C}$ overnight. Followed by incubation with a Cy3-conjugated goat anti-rabbit $\lg \mathrm{G}(\mathrm{H}+\mathrm{L})$ or FITC-conjugated goat anti-mouse $\lg \mathrm{G}(\mathrm{H}+\mathrm{L})$ for $1 \mathrm{~h}$. After washing with PBS thrice, the cells nuclei were counterstained with DAPI $(0.5 \mu \mathrm{g} / \mathrm{mL})$. The protein expression and nuclear translocation were

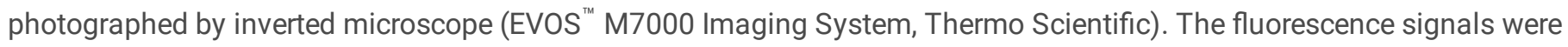
quantified with Image $\mathrm{J}$ software.

\subsection{Co-immunoprecipitation (Co-IP)}

After experimental treatments, proteins from GC-2spd(ts) cells were harvested by ice-cold IP Lysis (Thermo Fisher Scientific, USA). Co-IP assays were conducted using Pierce Co-IP Kit according to the manufacturer's instructions. Approximate $30 \mu \mathrm{g}$ of affinitypurified Nrf2 was incubated with aminoLink plus coupling resin. Next, protein mixture was added into the appropriate resin and the mixture rocking for overnight at $4^{\circ} \mathrm{C}$. Next day, $10 \mu \mathrm{L}$ of elution buffer was added into coupling resin, followed by centrifuging. Repeating this step for 4 times. Subsequently, the eluted proteins were boiled with $5 \times$ sample buffer and western blot analysis as descried in the above section.

\subsection{Nondenature PAGE (N-PAGE) analysis.}

As previously described (Wei et al., 2020), total protein was extracted from GC-2spd(ts) cells using ice-cold IP Lysis. Removing cell debris, $5 \times$ Native Sample Loading Buffer was added to the supernatant, which were resolved on $12 \%$ PAGE gels. The following steps were the same as western blot analysis.

\subsection{Statistical analysis}

The experimental results were presented as the means \pm S.E.M. All experiments represent at least 3 independent experiments. Statistical significance was analyzed by one-way ANOVA. $P<0.05$ was defined significant difference. 


\section{Results}

\subsection{Accumulation of nanoplastics in mouse spermatocyte-derived GC- 2 spd(ts) cells}

No fluorescent signal was observed in the control group after $24 \mathrm{~h}$. As expected, we found that $80 \mathrm{~nm}$ fluorescent NaPs could enter into GC-2spd(ts) cells by fluorescent inverted microscope for $24 \mathrm{~h}$ (Fig. 2). $\mathrm{H}_{2} \mathrm{~S}$ donor have been reported to prevent redox system imbalance via increasing Nrf2-medicated antioxidant defenses (Zhao et al., 2020). To investigate how microplastics stress influences redox system, we used Nrf2 activator, $\mathrm{H}_{2} \mathrm{~S}$ donor, to explore the role of $\mathrm{Nrf} 2$ in NaPs-induced reproductive toxicity in vitro. Here, we also found that NaHS pre-treatment did not reduce fluorescent signal in the presence of NaPs (Fig. 2).

\subsection{NaPs-induced oxidative stress via regulating Nrf2/Keap1 signaling in vitro}

At first, using immunofluorescence assay, we observed that NaPs decreased the fluorescence intensity of Nrf2, which was mainly located in the cytoplasm. By contrast, the view of immunofluorescence microscopy showed that NaHS treatment promoted Nrf2 expression and enhanced nuclear localization of Nrf2 in GC-2spd(ts) cells (Fig. 3A-B). On the other hand, our data showed that Nrf2 activation was necessary for NaHS to suppress NaPs-caused ROS burst (Supplementary Fig. 1). Moreover, western blotting showed that NaPs treatment significantly decreased the protein levels of Nrf2, HO-1, Nqo1 and increased the protein levels of Keap1 in GC-2spd(ts) cells, and these changes could be mitigated by NaHS pre-treatment (Fig. 3C-D). Next, Co-IP analysis showed that NaPs treatment did not change the interaction between Nrf2 and Keap1. However, pre-treatment with NaHS weakened the interaction between Keap1 and Nrf2 (Fig. 3E-F), indicating $\mathrm{H}_{2} \mathrm{~S}$ donor contribute to Nrf2 translocated to the nucleus and induced transcription of antiantioxidant genes. Through flow cytometry assays, we found that NaPs treatment significantly triggered the generation of endogenous ROS (Fig. 3A-B). By contrast, the over-production of endogenous ROS induced by NaPs was also decreased after improving Nrf2/Keap1 signaling (Fig. 3G-H). Above results suggest that loss of Nrf2 was active regulator for oxidative stress caused by NaPs in GC-2spd(ts) cells.

\subsection{NaPs-induced reproductive toxicity was mediated by ROS-dependent mitochondrial apoptosis in vitro}

Next, we attempted to explore whether oxidative stress posed by NaPs exposure associated mitochondrial apoptosis in GC2 spd(ts) cells. Flow cytometry analysis revealed that NaPs significantly increased the percentage of apoptosis cells (Fig. 4A-B). We also demonstrated that suppression of excessive ROS by NaHS pre-treatment significantly diminished the percentage of apoptosis cells in the presence of NaPs (Fig. 4A-B). Next, found that NaPs significantly caused a noteworthy dissipation of the MMP, decreased by almost 9-fold, which can be reversed by suppression of excessive ROS (Fig. 4C-D). Meanwhile, the view of fluorescent inverted microscope showed that the green fluorescence intensity was significantly reduced after NaPs exposure. Whereas, suppression of excessive ROS remarkably inhibited green fluorescence intensity and increased red fluorescence intensity in GC-2spd(ts) cells (Fig. 4E). Meanwhile, we also demonstrated that the regulation of Nrf2 signaling was one of the mechanisms in NaPs-induced reproductive toxicity (Supplementary Fig. 2). Collectively, these results indicate that NaPs-induced mitochondrial apoptosis was mediated by ROS in mice spermatocyte cells.

\subsection{Excessive ROS mediated NaPs-induced excessive autophagy in GC- 2spd(ts) cells}

Accumulating evidence indicates that Nrf2 signaling regulates the intrinsic autophagy impairment (Zhang et al., 2017; Wang et al., 2020). We then investigated whether autophagy was associated with NaPs-induced dysregulation of Nrf2 in vitro. At first, western blotting analysis revealed that NaPs significantly promoted the the expression of autophagy biomarker LC3-II, p62 and reduced expression of mTORC (Fig. 5A-D), suggesting that NaPs could promote excessive autophagy via regulating mTORC signaling in GC-2spd(ts) cells. In contrast, suppression of excessive ROS by NaHS abrogated the regulatory effects of NaPs on LC3-II, p62 and mTORC protein expressions (Fig. 5A-D). In accordance with these results, immunofluorescence assays confirmed that NaPs incubation up-regulated LC3-II protein levels, as evidenced by the enhance red fluorescence. Similarly, NaPs incubation increased 
LC3-II protein levels, which was normalized by NaHS treatment (Fig. 5E-F). These data suggested that excessive ROS triggered excessive autophagy and thus involved in NaPs-triggered reproductive toxicity in vitro.

\subsection{PKM2 signaling was involved in NaPs-triggered reproductive toxicity in vitro}

Normal rate-limiting enzyme (PKM2, GLU and HKII) of aerobic glycolysis levels are necessary for energy production and cellular physiology. Additionally, accumulating evidence has demonstrated that PKM2, as a transcriptional coactivator of Nrf2, generates sufficient GSH for ROS detoxification (Wei et al., 2020). Herein, western blotting analysis revealed that the expressions of GLU and HKII were not significantly influenced by NaPs incubation (Fig. 6A-B). In contrast, we found that the expression of PKM2 was increased in GC-2spd(ts) cells after NaPs treatment. Meanwhile, we pretreated cells with NaHS to activate Nrf2 signaling. We found that the altered gene expression of PKM2 was reversed by the activate Nrf2 signaling (Fig. 6A-B). Immunofluorescence assays further confirmed that NaPs incubation reduced PKM2 protein level, as evidenced by the weaker fluorescence. Consistent with the western blotting results, immunofluorescence assays also demonstrated that PKM2 nuclear translocation was enhanced by the combined treatment of NaPs and NaHS (Fig. 6C-D). Hence, these data suggested that NaPs might switch specific biological functions of PKM2 relying on Nrf2 signaling.

\subsection{NaPs-altered dimerize PKM2 expressions was associated with Nrf2 signaling}

PKM2 exists in the form of tetramers and dimers with different biological activities. Next, we found that dimerize PKM2 expression was significantly reduced by NaPs incubation (Fig. 7A-B). Meanwhile, we also observed that NaPs treatment mildly promoted the expression of tetrameric PKM2 (Fig. 7A and D). In contrast, NaPs-altered dimerize and tetrameric PKM2 expressions were significantly mitigated by NaHS co-treatment (Fig. 7A-C). As we expected, Co-IP analysis proved that PKM2 directly interacted with Nrf2 (Fig. 7C-D). These data indicated that NaPs could regulate dimerize PKM2 expression via Nrf2 signaling.

\section{Discussion}

In the present study, we illustrated the molecular mechanism of the NaPs-induced reproductive toxicity in vitro. We confirmed that NaPs caused ROS burst associated with Nrf2/Keap1 signaling. Moreover, current results also suggested dysregulation of Nrf2 signaling and dimerize PKM2 involved in NaPs induced mitochondrial apoptosis and excessive autophagy in vitro. Another novel finding was that enhanced interaction between PKM2 and Nrf2 might be a crucial regulator of targeted prevention of NaPsinduced reproductive toxicity. The work revealed the detailed underlying mechanism about the NaPs-induced reproductive toxicity, and extended our recognition on the relationship between plastic and reproductive disease risk.

In general, the Insufficient antioxidant enzymes may be the main cause and a key event in the development of declining semen quality. Herein, we demonstrated that NaPs could led to excessive ROS generation in GC-2spd(ts) cells, which may be due to the fact that the higher mass/surface ratio of NaPs enable the translocation of free radicals through membranes (Lei et al., 2018). In addition to the absorption mechanism of free radicals, NaPs-induced oxidative stress may also be explained by the depletion of antioxidant enzymes. Consistent with the fact, we also found that NaPs can inhibit Nrf2-mediated antioxidant capacity, indicating NaPs disturbed proper redox homeostasis in mice spermatocyte cells. Consistently, an in vivo study on testicular toxicity showed that polystyrene microplastics could induce the decline of sperm quality via decreasing the protein expressions of Nrf2 and HO-1 (Hou et al., 2021b). Furthermore, previous research also demonstrated that microplastics could increase oxdative stress via inhibiting nuclear accumulation of Nrf2 protein, which supports our data (Li et al., 2021b). Similarly, plastic additives could increase oxidative stress in testes via promoting m6A modification of Nrf2 mRNA (Zhao et al., 2020). Alternatively, this may be due to the fact that NaPs could induce mitochondrial membrane dysfunction, subsequently causing ROS burst via one-electron carriers (Zhao et al., 2020).

Because Nrf2 is captured by Keap1 and constantly degraded via the ubiquitin-proteasome pathway in cytoplasm, we speculated that modifying the binding of Nrf2-Keap1 might provide a way to increase antioxidant protection. Previous study has demonstrated that $\mathrm{H}_{2} \mathrm{~S}$ could increase the glutathione contents and alleviate oxidative stress via S-sulfhydration of Keap1 (Yang et al., 2013). Furthermore, administration of exogenous $\mathrm{H}_{2} \mathrm{~S}$ decreased the interaction between Keap1 and Nrf2 via S-sulfhydration 
of Keap1 at Cys151 in liver injury model (Zhao et al., 2021). In the current study, immunoprecipitation assay reveal that NaHS pretreatment reduced the binding of Keap1 to Nrf2 after NaPs exposure in GC-2spd(ts) cells. Additionally, we also found that NaHS could promote nuclear accumulation of Nrf2. These results suggest that NaHS could induced the dissociation of Nrf2 from Keap1, promote the nuclear translocation of $\mathrm{Nrf2}$, and subsequently enhance the antioxidant ability. On the other hand, we also confirmed that NaPs-induced oxidative stress in GC-2spd(ts) cells via downregulating Nrf2 signaling. In contrast, increased Nrf2 translocation by NaHS could suppress NaPs-caused excessive ROS. This finding further elucidated that NaPs-induced redox imbalance is relied on the dysregulation of Nrf2 signaling.

An important and novel finding in this study was that NaPs decreased the expression of PKM2 dimer. It has been reported that the dimeric PKM2 in the nucleus acts as the a transcription factor coactivator to regulate gene expression, and attaches to the outer membrane of mitochondrial to maintain mitochondrial function (Christofk et al., 2008). Interestingly, the dimerization of PKM2 has been reported to interact with and activate Nrf2, thereby facilitating GSH synthesis (Wei et al., 2020b). Consistently, Co-IP analysis in this study showed that enhanced the binding activity of Nrf2 with PKM2 could rescue NaPs-triggered mitochondrial apoptosis in GC-2spd(ts) cells. This findings suggested that enhanced interaction between PKM2 and Nrf2 might obtain the advantage of transcription factors to alleviate the ROS burst (Gao et al., 2020). However, further studies should be conducted to confirm the underlying mechanisms.

Interestingly, we found that NaPs exposure could increase autophagy levels, evidenced by the up-regulated expression of LC3II and p62. One mechanism could be that NaPs might damage the lysosomal compartment and mitochondria, or change the cell cytoskeleton, thereby resulting in the blockade of autophagosome-lysosome fusion (Stern et al., 2012). Alternatively, perhaps this is due to the mechanism that NaPs could result in lysosomal oxidative stress and alkalization, subsequently inducing osmotic swelling and detergent-like disruption of the lysosomal membrane, which in turn leads to lysosomal membrane permeabilization (Xia et al., 2008; Ma et al., 2011). In this context, it seems that NaPs could induce lysosomal dysfunction, which contributed to accumulation of autophagosomes and ubiquitinated protein aggregates. Notably, we also found that improving Nrf2 translocation via pro-treated with NaHS counteracted NaPs-induced autophagy in GC-2spd(ts) cells. These results are supported by previous studies demonstrating that activating Nrf2-meidated antioxidant capacity inhibited autophagic cell death in the spleen (Zhao et al., 2021). It is known that excessive ROS burst can lead to cellular biomolecules damage and organelle dysfunction, thereby facilitating accumulation of protein damage products (Zhang et., 2013). Furthermore, excessive ROS serve as signaling molecules to oxidize mTORC, thereby promoting autophagy (Li et al., 2021). In combination with the mechanisms of ROS-triggered autophagy, our data suggest that NaPs-induced excessive autophagy might attribute to loss of Nrf2 signaling in GC-2spd(ts) cells. This point is supported by previous study (Liu et al., 2016).

\section{Conclusion}

Our research demonstrated that NaPs-induced reproductive toxicity was mediated by ROS-dependent mitochondrial apoptosis and autophagy in vitro. Suppression of ROS burst by Nrf2-mediated anti-oxidant defence with the regulation of PKM2 signaling could mitigate NaPs-mediated reproductive toxicity in vitro. Moreover, increasing interaction between PKM2 and Nrf2, and this cooperation might alleviate the NaPs-mediated reproductive toxicity in vitro (Fig. 8). Thus, interaction of PKM2 with Nrf2 signaling are essential process of NaPs-induced reproductive toxicity via disturbing tolerance to environmental stress. This stud provides a new perspective for understanding microplastics-induced reproductive toxicity mechanisms.

\section{Declarations}

\section{Data Availability}

All supporting data are included within the main article and supplementary files.

\section{Funding}

The current work was supported by the National Natural Science Foundation of China (NO. 81773478) and Natural Science Foundation of Hunan Province, China (NO. 2020JJ3052) and Postgraduate Independent Exploration and Innovation Project of Central South University (160171001). 


\section{Contributions}

Siwen Li: Investigation, Writing-original draft, Writing-review \& editing, Data curation. Yu Ma and Shuzi Ye: Software, Formal analysis. Siwen Li, Ying Su and Die Hu: Resources and Formal analysis. Fang Xiao: Conceptualization, Funding acquisition.

\section{Ethics declarations}

\section{Conflict of interest}

The authors declare that they have no conflict of interest.

\section{References}

1. Amereh F, Babaei M, Eslami A, Fazelipour S, Rafiee M (2020) The emerging risk of exposure to nano(micro)plastics on endocrine disturbance and reproductive toxicity: From a hypothetical scenario to a global public health challenge. Environ Pollut 261:114158. https://doi.org/10.1016/j.envpol.2020.114158

2. Christofk HR, Vander HM, Harris MH, Ramanathan A, Gerszten RE, Wei R, Fleming MD, Schreiber SL, Cantley LC (2008) The M2 splice isoform of pyruvate kinase is important for cancer metabolism and tumour growth. Nature 452:230-233. https://doi.org/10.1038/nature06734

3. Da CAA, Malafaia G (2021) Microplastic ingestion induces behavioral disorders in mice: A preliminary study on the trophic transfer effects via tadpoles and fish. J Hazard Mater 401:123263. https://doi.org/10.1016/j.jhazmat.2020.123263

4. Deng Y, Yan Z, Shen R, Huang Y, Ren H, Zhang Y (2021) Enhanced reproductive toxicities induced by phthalates contaminated microplastics in male mice (Mus musculus). J Hazard Mater 406:124644. https://doi.org/10.1016/j.jhazmat.2020.124644

5. Deng Y, Zhang Y, Lemos B, Ren H (2017) Tissue accumulation of microplastics in mice and biomarker responses suggest widespread health risks of exposure. Sci Rep 7:46687. https://doi.org/10.1038/srep46687

6. Dong T, Yan Y, Chai H, Chen S, Xiong X, Sun D, Yu Y, Deng L, Cheng F (2015) Pyruvate kinase M2 affects liver cancer cell behavior through up-regulation of HIF-1alpha and Bcl-xL in culture. Biomed Pharmacother 69:277-284.

https://doi.org/10.1016/j.biopha.2014.12.010

7. Gao XH, Li L, Parisien M, Wu J, Bederman I, Gao Z, Krokowski D, Chirieleison SM, Abbott D, Wang B, Arvan P, Cameron M, Chance M, Willard B, Hatzoglou M (2020) Discovery of a redox thiol switch: implications for cellular energy metabolism. Mol Cell Proteomics 19:852-870. https://doi.org/10.1074/mcp.RA119.001910

8. Geyer R, Jambeck JR, Law KL (2017) Production, use, and fate of all plastics ever made. Sci Adv 3:e1700782. https://doi.org/10.1126/sciadv.1700782

9. Hou B, Wang F, Liu T, Wang Z (2021) Reproductive toxicity of polystyrene microplastics: In vivo experimental study on testicular toxicity in mice. J Hazard Mater 405:124028. https://doi.org/10.1016/j.jhazmat.2020.124028

10. Jeong CB, Won EJ, Kang HM, Lee MC, Hwang DS, Hwang UK, Zhou B, Souissi S, Lee SJ, Lee JS (2016) Microplastic SizeDependent Toxicity, Oxidative Stress Induction, and p-JNK and p-p38 Activation in the Monogonont Rotifer (Brachionus koreanus). Environ Sci Technol 50:8849-8857. https://doi.org/10.1021/acs.est.6b01441

11. Jin H, Ma T, Sha X, Liu Z, Zhou Y, Meng X, Chen Y, Han X, Ding J (2021) Polystyrene microplastics induced male reproductive toxicity in mice. J Hazard Mater 401:123430. https://doi.org/10.1016/j.jhazmat.2020.123430

12. Lei L, Liu M, Yang S, Lu S, Hu J, Cao C, Xie B, Shi H, He D (2018) Polystyrene (nano)microplastics cause size-dependent neurotoxicity, oxidative damage and other adverse effects in Caenorhabditis elegans. Environmental Science Nano, 10-1039. https://doi.org/10.1039/c8en00412a

13. Li S, Ma Y, Ye S, Hu D, Xiao F (2021) ERK/P38/ROS burst responses to environmentally relevant concentrations of diphenyl phosphate-evoked neutrophil extracellular traps formation: Assessing the role of autophagy. J Hazard Mater 14:126758. https://doi.org/10.1016/j.jhazmat.2021.126758

14. Li S, Ma Y, Ye S, Ta Ng S, Liang N, Liang Y, Xiao F (2021) Polystyrene microplastics trigger hepatocyte apoptosis and abnormal glycolytic flux via ROS-driven calcium overload. J Hazard Mater 417:126025.

https://doi.org/10.1016/j.jhazmat.2021.126025

Page $8 / 18$ 
15. Li S, Shi M, Wang Y, Xiao Y, Cai D, Xiao F (2021a) Keap1-Nrf2 pathway up-regulation via hydrogen sulfide mitigates polystyrene microplastics induced-hepatotoxic effects. J Hazard Mater 402:123933.

https://doi.org/10.1016/j.jhazmat.2020.123933

16. Liu J, Wu J, Sun A, Sun Y, Yu X, Liu N (2016) Hydrogen sulfide decreases high glucose/palmitate-induced autophagy in endothelial cells by the Nrf2-ROS-AMPK signaling pathway. Cell Bioscience 6:33. https://doi.org/10.1186/s13578-016-0099-1

17. Li XN, Yang SQ, Li M, Li XS, Tian Q, Xiao F, Tang YY, Kang X, Wang CY, Zou W, Zhang P, Tang XQ (2021) Formaldehyde induces ferroptosis in hippocampal neuronal cells by upregulation of the Warburg effect. Toxicology 448:152650. https://doi.org/10.1016/j.tox.2020.152650

18. Li Y, Feng YF, Liu XT, Li YC, Zhu HM, Sun MR, Li P, Liu B, Yang H (2021) Songorine promotes cardiac mitochondrial biogenesis via Nrf2 induction during sepsis. Redox Biol 38:101771. https://doi.org/10.1016/j.redox.2020.101771

19. Li Z, Zhu S, Liu Q, Wei J, Jin Y, Wang X, Zhang L (2020) Polystyrene microplastics cause cardiac fibrosis by activating Wnt/beta-catenin signaling pathway and promoting cardiomyocyte apoptosis in rats. Environ Pollut 265:115025. https://doi.org/10.1016/j.envpol.2020.115025

20. Luo W, Hu H, Chang R, Zhong J, Knabel M, O'Meally R, Cole RN, Pandey A, Semenza GL (2011) Pyruvate kinase M2 is a PHD3stimulated coactivator for hypoxia-inducible factor 1. Cell 145:732-744. https://doi.org/10.1016/j.cell.2011.03.054

21. Ma X, Wu Y, Jin S, Tian Y, Zhang X, Zhao Y, Yu L, Liang XJ (2011) Gold nanoparticles induce autophagosome accumulation through size-dependent nanoparticle uptake and lysosome impairment. ACS NANO 5:8629-8639.

https://doi.org/10.1021/nn202155y

22. Plastics, Europe (2018) Plastics-the facts 2018. Available online. http://www.plasticseurope.org/application/files/5715/1717/4180/Plastics_the_facts_2017_FINAL_for_website_one_page.pdf

23. Rahman A, Sarkar A, Yadav OP, Achari G, Slobodni KJ (2021) Potential human health risks due to environmental exposure to nano- and microplastics and knowledge gaps: a scoping review. Science of The Total Environment 757:143872. https://doi.org/10.1016/j.scitotenv.2020.143872

24. Ren L, Liu J, Zhang J, Wang J, Wei J, Li Y, Guo C, Sun Z, Zhou X (2019) Silica nanoparticles induce spermatocyte cell autophagy through microRNA-494 targeting AKT in GC-2spd cells. Environ Pollut 255:113172. https://doi.org/10.1016/j.envpol.2019.113172

25. Santini SJ, Cordone V, Falone S, Mijit M, Tatone C, Amicarelli F, Di Emidio G (2018) Role of Mitochondria in the Oxidative Stress Induced by Electromagnetic Fields: Focus on Reproductive Systems. Oxid Med. Cell. Longev. 2018, 5076271. https://doi.org/10.1155/2018/5076271

26. Sedha S, Kumar S, Shukla S (2015) Role of Oxidative Stress in Male Reproductive Dysfunctions with Reference to Phthalate Compounds. Urol J 12:2304-2316. https://doi.org/10.22037/uj.v12i5.3009

27. Stern ST, Adiseshaiah PP, Crist RM (2012) Autophagy and lysosomal dysfunction as emerging mechanisms of nanomaterial toxicity. Part Fibre Toxicol 9:20. https://doi.org/10.1186/1743-8977-9-20

28. Van Sebille E, Wilcox C, Lebreton L, Maximenko N (2015) A global inventory of small floating plastic debris. Environ Res Lett 10:124006. https://doi.org/10.1088/1748-9326/10/12/124006

29. Wang Y, Zhao H, Guo M, Fei D, Zhang L, Xing M (2020) Targeting the miR-122/PKM2 autophagy axis relieves arsenic stress. J Hazard Mater 383:121217. https://doi.org/10.1016/j.jhazmat.2019.121217

30. Wei Y, Lu M, Mei M, Wang H, Han Z, Chen M, Yao H, Song N, Ding X, Ding J, Xiao M, Hu G (2020) Pyridoxine induces glutathione synthesis via PKM2-mediated Nrf2 transactivation and confers neuroprotection. Nat Commun 11:941. https://doi.org/10.1038/s41467-020-14788-x

31. Wright SL, Kelly FJ (2017) Plastic and Human Health: A Micro Issue? Environ Sci Technol 51:6634. https://doi.org/10.1021/acs.est.7b00423

32. Xia T, Kovochich M, Liong M, Zink JI, Nel AE (2008) Cationic polystyrene nanosphere toxicity depends on cell-specific endocytic and mitochondrial injury pathways. ACS NANO 2:85-96. https://doi.org/10.1021/nn700256c

33. Xie X, Deng T, Duan J, Xie J, Yuan J, Chen M (2020) Exposure to polystyrene microplastics causes reproductive toxicity through oxidative stress and activation of the p38 MAPK signaling pathway. Ecotoxicol Environ Saf 190:110133. 
https://doi.org/10.1016/j.ecoenv.2019.110133

34. Yang G, Zhao K, Ju Y, Mani S, Cao Q, Puukila S, Khaper N, Wu L, Wang R (2013) Hydrogen sulfide protects against cellular senescence via S-sulfhydration of Keap1 and activation of Nrf2. Antioxid Redox Signal 18:1906-1919.

https://doi.org/10.1089/ars.2012.4645

35. Yin K, Wang Y, Zhao H, Wang D, Guo M, Mu M et al. A comparative review of microplastics and nanoplastics: toxicity hazards on digestive, reproductive and nervous system. Science of The Total Environment, 774: 145758.

https://doi.org/10.1016/j.scitotenv.2021.145758

36. Zhang DD, Chapman E (2020) The role of natural products in revealing Nrf2 function. Nat Prod Rep 37:797-826. https://doi.org/10.1039/C9NP00061E

37. Zhang HS, Zhang ZG, Zhou Z, Du GY, Li H, Yu XY, Huang YH (2017) PKM2-mediated inhibition of autophagy facilitates Tat's inducing HIV-1 transactivation. Arch Biochem Biophys 625-626:17-23. https://doi.org/10.1016/j.abb.2017.05.018

38. Zhang J, Bao X, Zhang M, Zhu Z, Zhou L, Chen Q, Zhang Q, Ma B (2019) MitoQ ameliorates testis injury from oxidative attack by repairing mitochondria and promoting the Keap1-Nrf2 pathway. Toxicol Appl Pharmacol 370:78-92.

https://doi.org/10.1016/j.taap.2019.03.001

39. Zhao G, Qi B, Wang Y, Li X, Li Q, Tang X, Wang X, Wu C (2021) Antagonizing effects of curcumin against mercury-induced autophagic death and trace elements disorder by regulating PI3K/AKT and Nrf2 pathway in the spleen. Ecotoxicol Environ Saf 222:112529. https://doi.org/10.1016/j.ecoenv.2021.112529

40. Zhao S, Song T, Gu Y, Zhang Y, Cao S, Miao Q, Zhang X, Chen H, Gao Y, Zhang L, Han Y, Wang H, Pu J, Xie L, Ji Y (2021) Hydrogen sulfide alleviates Liver injury through the S-sulfhydrated-kelch-Like ECH-associated protein 1/Nuclear erythroid 2related factor 2/low-Density lipoprotein receptor-related protein 1 pathway. Hepatology 73:282-302. https://doi.org/10.1002/hep.31247

41. Zhao TX, Wang JK, Shen LJ, Long CL, Liu B, Wei Y, Han LD, Wei YX, Wu SD, Wei GH (2020) Increased m6A RNA modification is related to the inhibition of the Nrf2-mediated antioxidant response in di-(2-ethylhexyl) phthalate-induced prepubertal testicular injury. Environ. Pollut. 259: 113911. https://doi.org/

42. Zheng D, Jiang Y, Qu C, Yuan H, Hu K, He L, Chen P, Li J, Tu M, Lin L, Chen H, Lin Z, Lin W, Fan J, Cheng G, Hong J (2020) Pyruvate Kinase M2 Tetramerization Protects against Hepatic Stellate Cell Activation and Liver Fibrosis. Am J Pathol 190:2267-2281. https://doi.org/10.1016/j.ajpath.2020.08.002

\section{Figures}



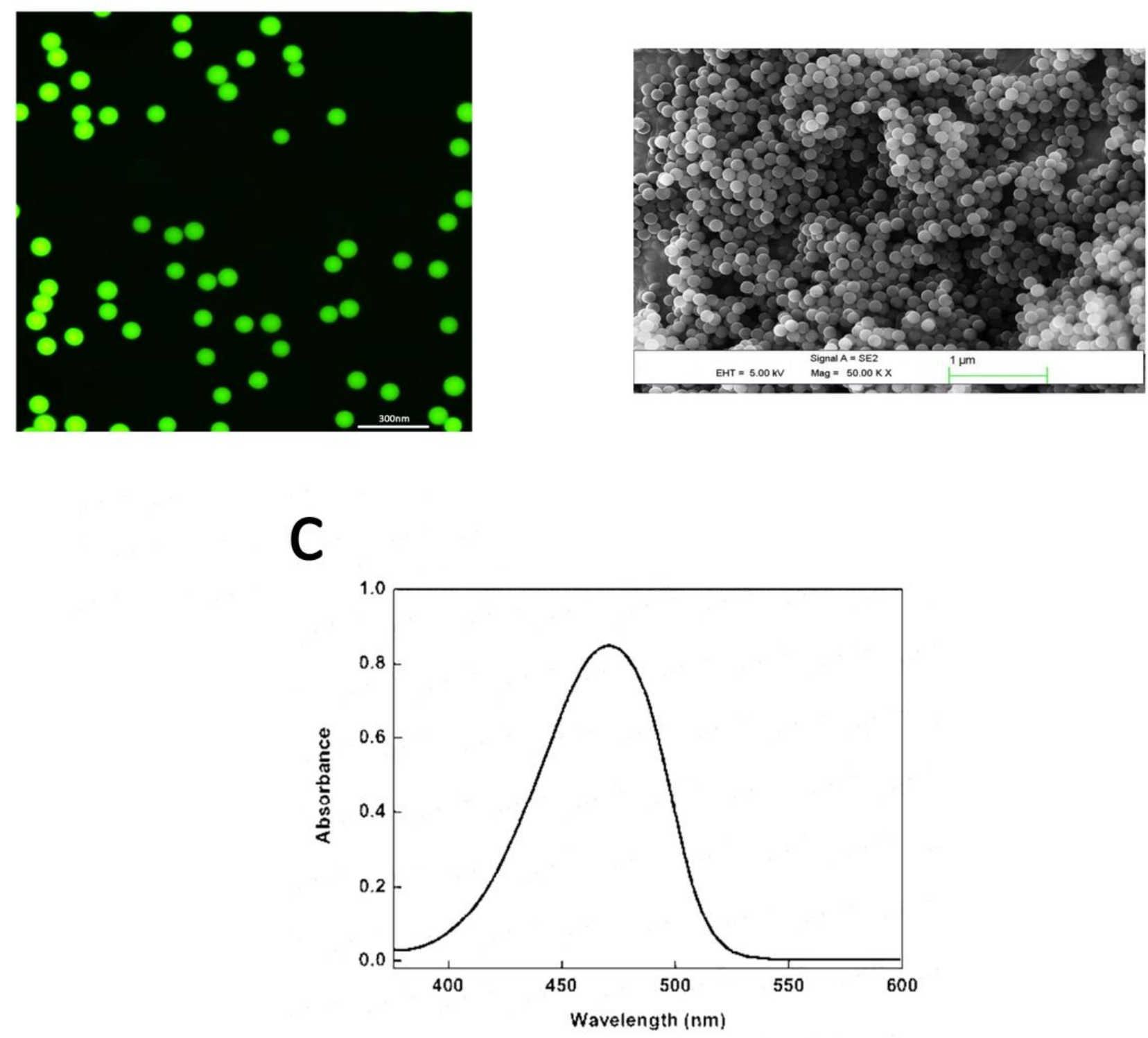

Figure 1

Characteristics and accumulation of nanoplastics. (A) The fluorescence microcopy images of nanoplastics used in this study. (B) Scanning electron microscope image of the morphology of nanoplastics. (C) UV-vis absorption spectra analysis. 

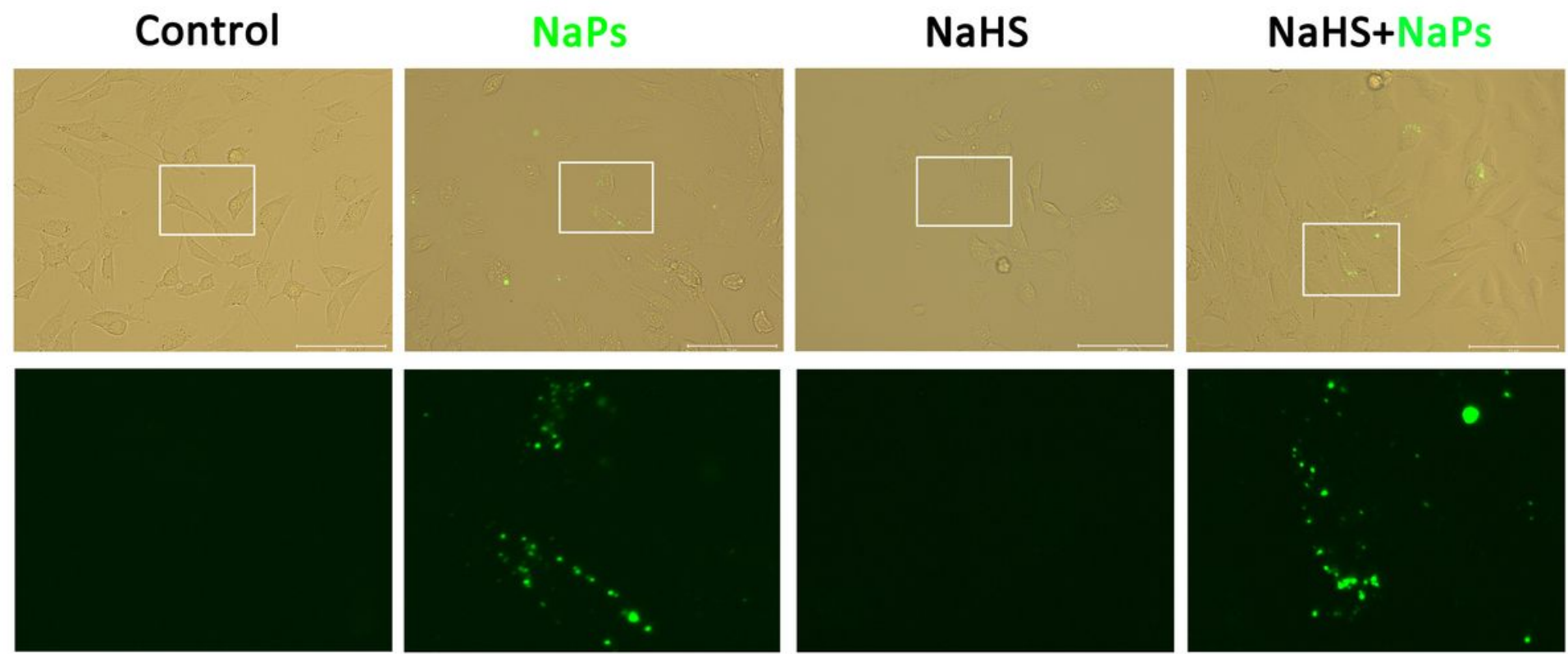

Figure 2

Entering of nanoplastics into mouse spermatocyte-derived GC-2spd(ts) cells after exposure for $24 \mathrm{~h}$. 
A
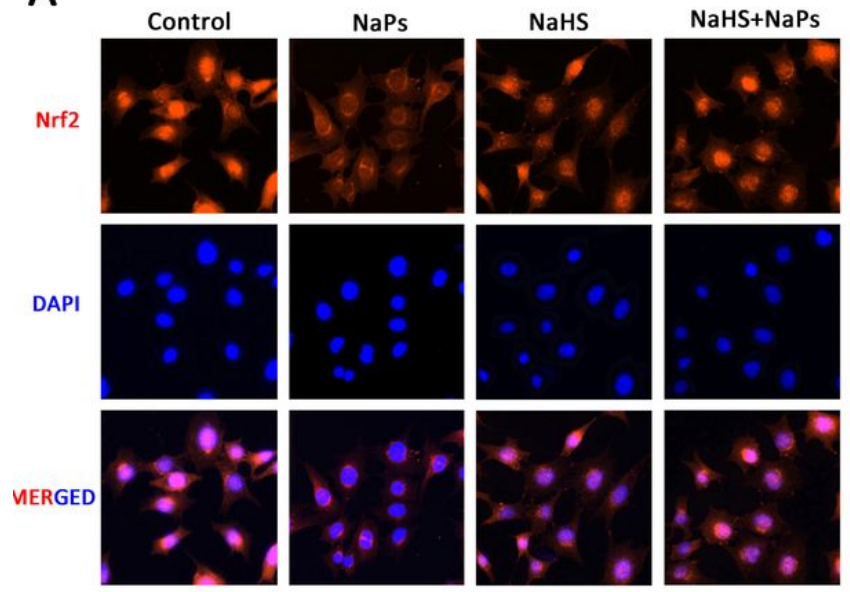

B

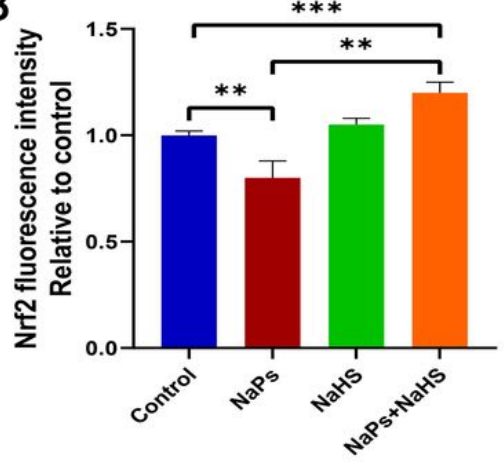

E

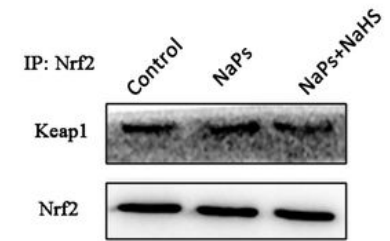

F

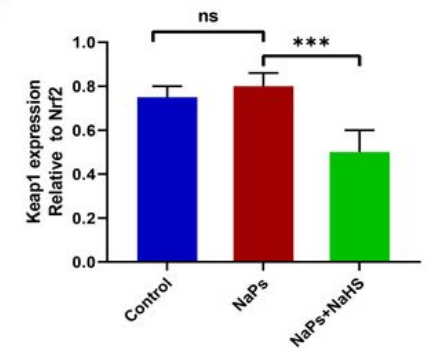

G

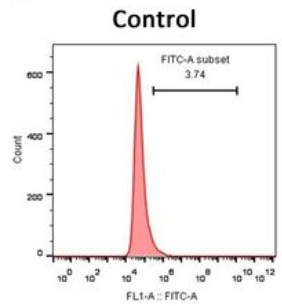

D

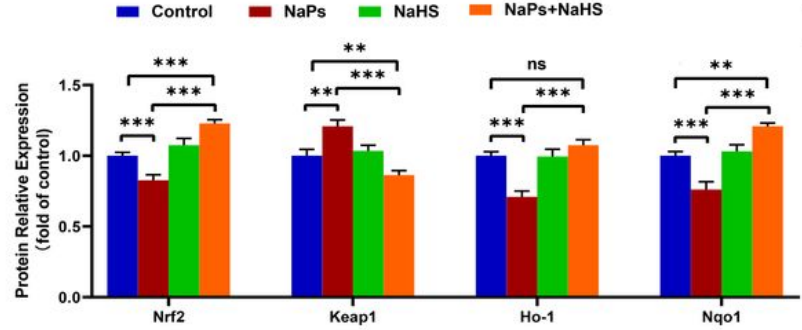

H

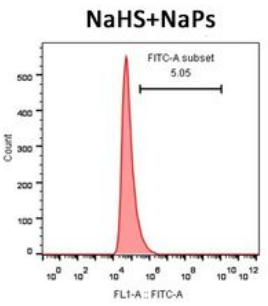

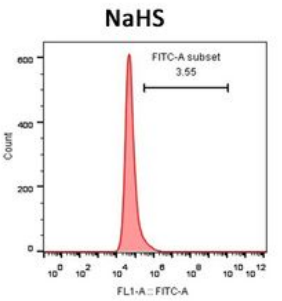

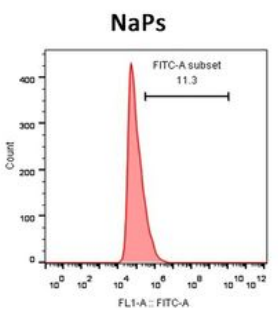

.

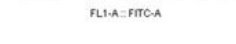

Ho-1

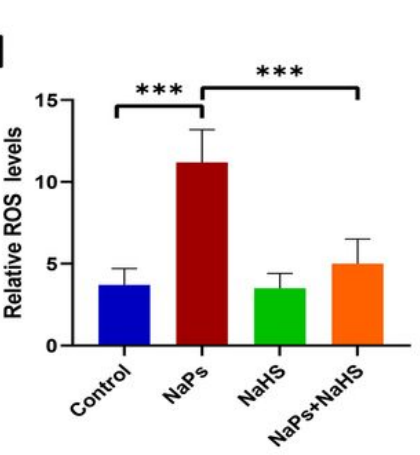

Figure 3

Improving Nrf2/Keap1 signaling combated NaPs-induced oxidative stress in the GC-2spd(ts) cells. (A) flow cytometry was used to identify intracellular ROS levels after loading with $10 \mu \mathrm{M}$ DCF-DA. (B) ROS levels were quantitated using the FlowJo software. (C) Nrf2 expression and location were confirmed by immunofluorescence analysis. (D) Statistical analysis of the fluorescence intensity of Nrf2 after treatment. (E) Western blotting was performed to determine Nrf2, Keap1, HO-1 and Nqo1 proteins expressions. (F) The relative protein expressions were evaluated by Image J software. (G) Co-IP analyses were performed to detect

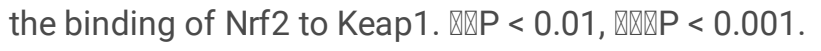


A
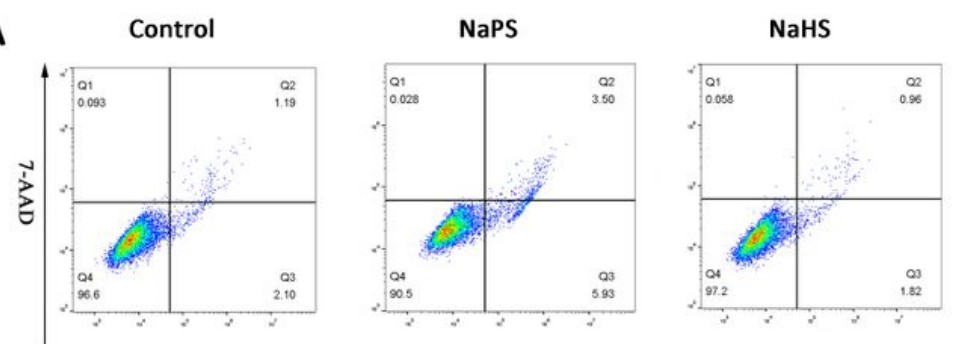

Annexin V
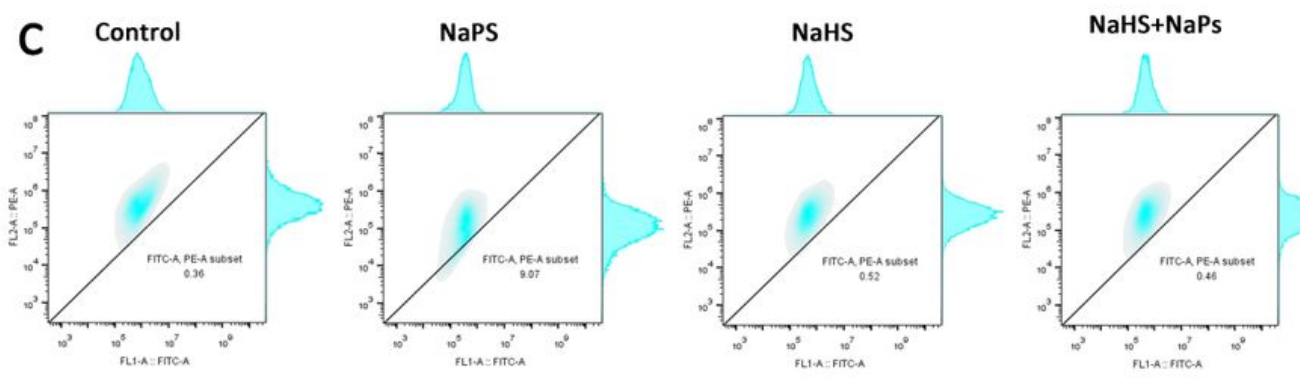

B

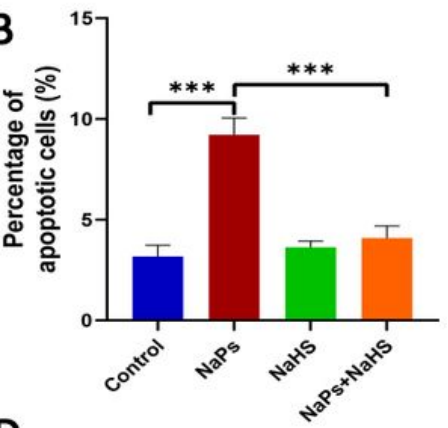

D

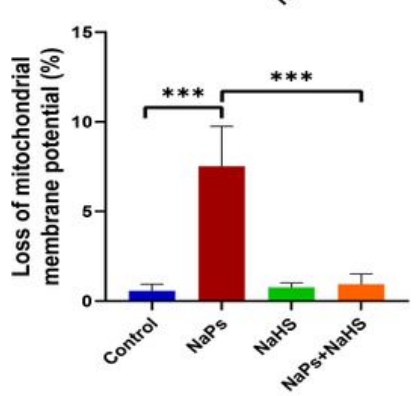

E

Control
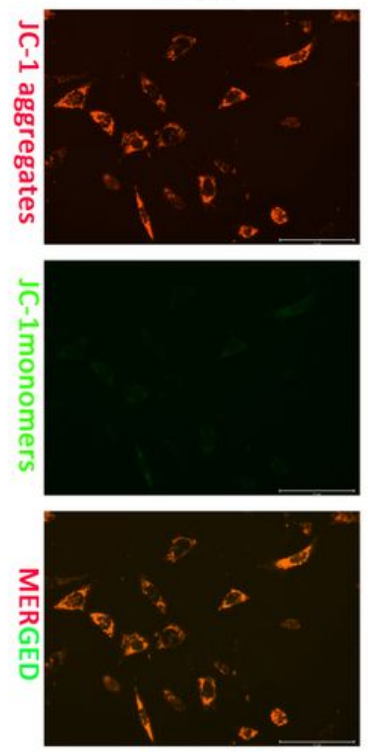

NaPS
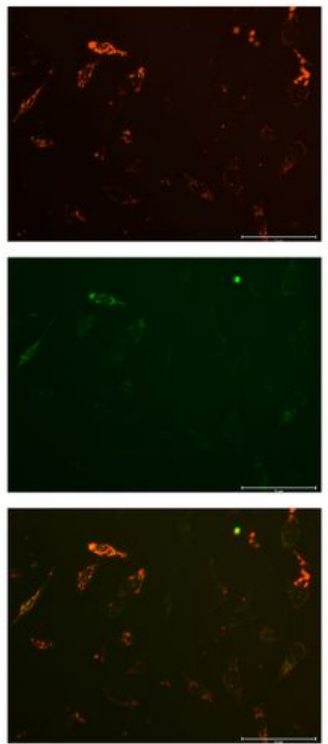
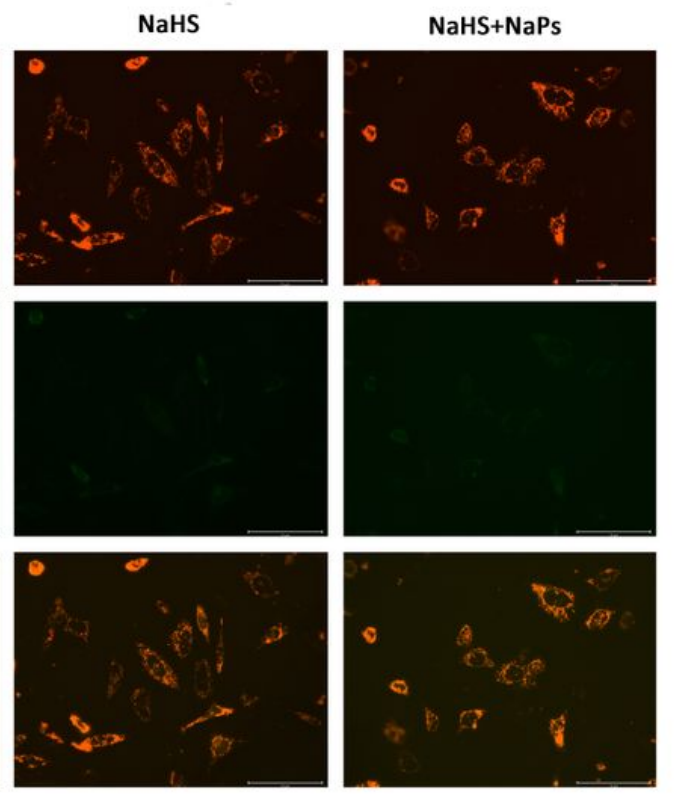

Figure 4

Suppression of excessive ROS suppressed NaPs-induced mitochondrial apoptosis. (A) Apoptosis was measured by the Annexin VPE/7-AAD double staining assay by flow cytometry analysis. (B) The proportion of apoptotic cells was analyzed using FlowJo software. (C) MMP were measured using JC-1 staining. (D) The quantification of MMP depolarization after NaHS or/and NaPs treatment. (E) Fluorescence merged images after JC-1 staining. captured by inverted fluorescence microscope. Scale bar $=20 \mu \mathrm{m}$. $\star * \star * P<0.001$. 

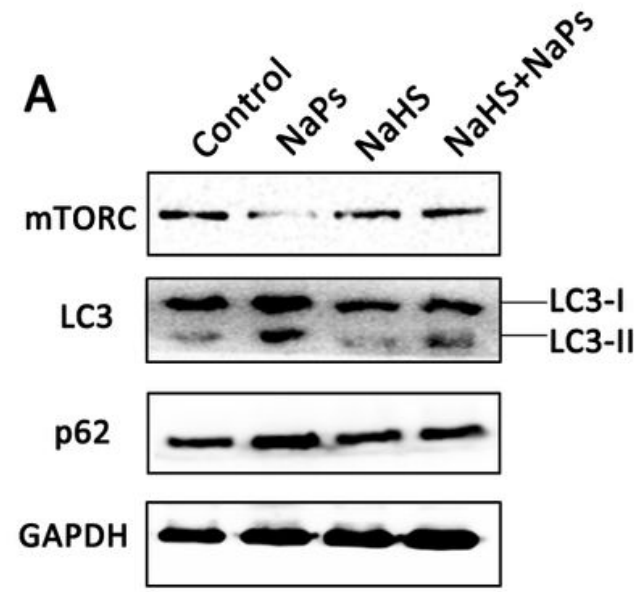

D
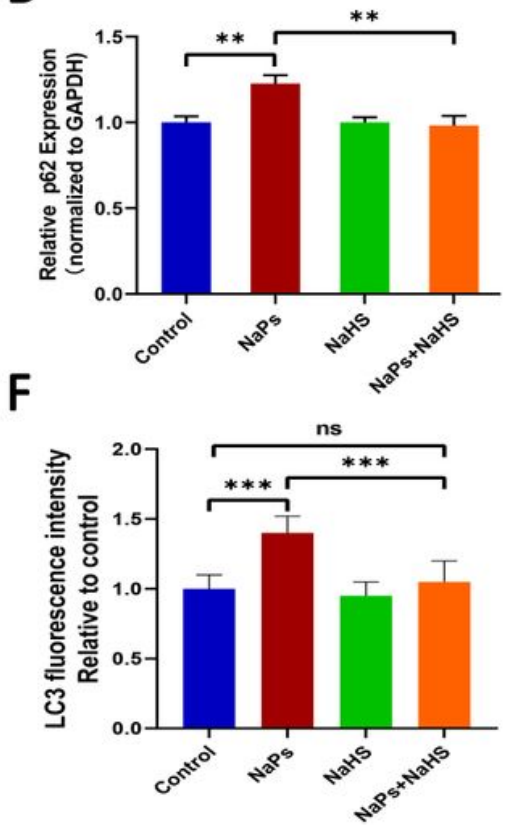

B

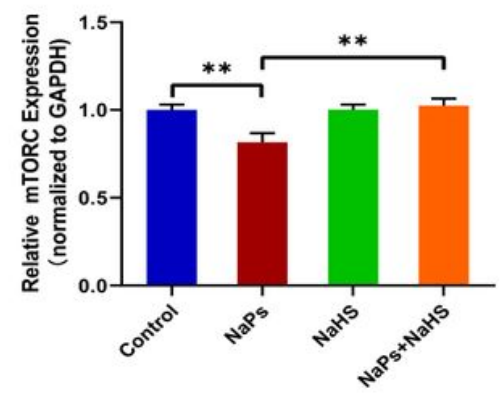

E

LC3-II
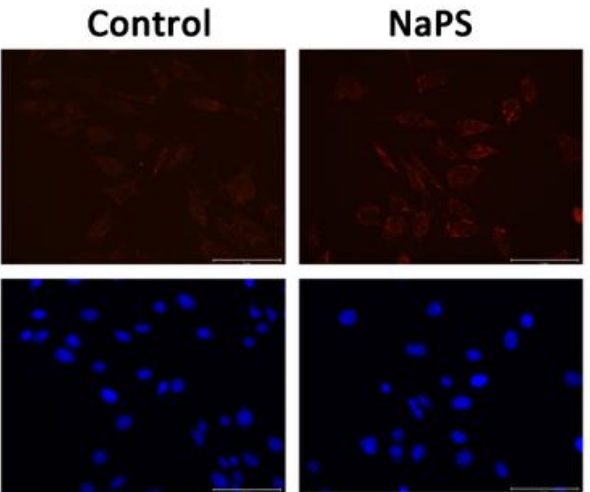

MERGED
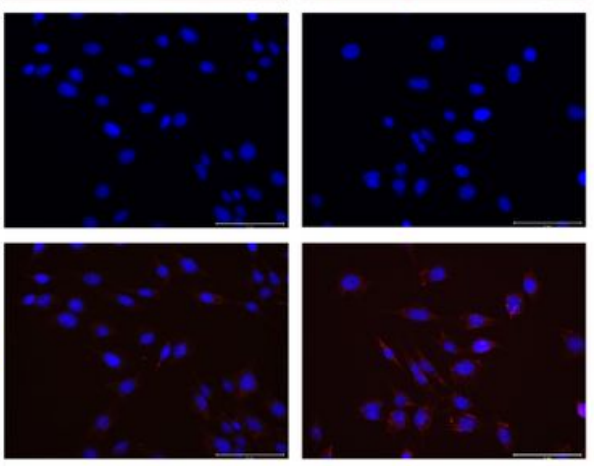

DAPI

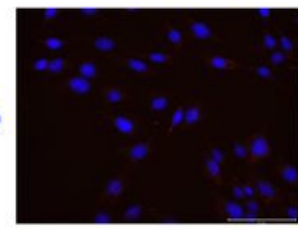

C
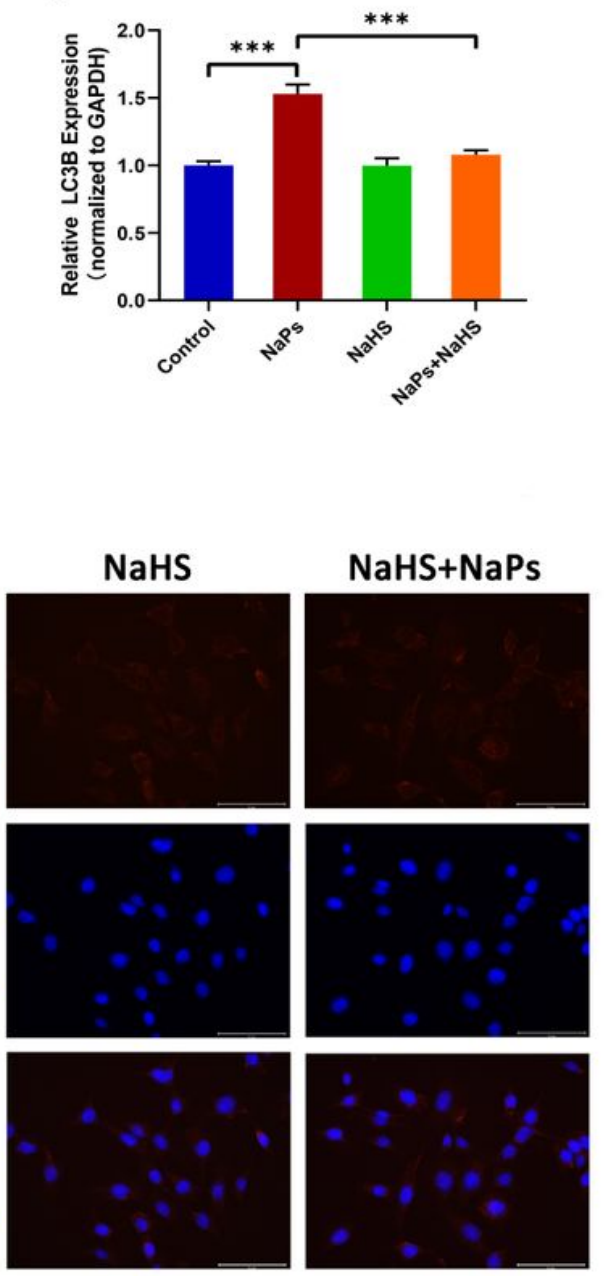

\section{Figure 5}

Suppression of excessive ROS alleviated excessive autophagy induced by NaPs in GC-2spd(ts) cells. (A) Western blotting was performed to determine mTORC, p62 and LC3-I/II proteins expressions. (B-D) The relative protein expressions were evaluated by Image J software. (E) LC3-II expression was confirmed by immunofluorescence analysis. (F) Statistical analysis of the

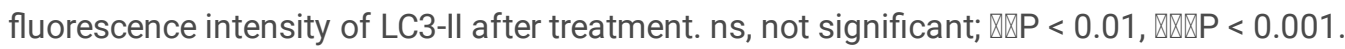



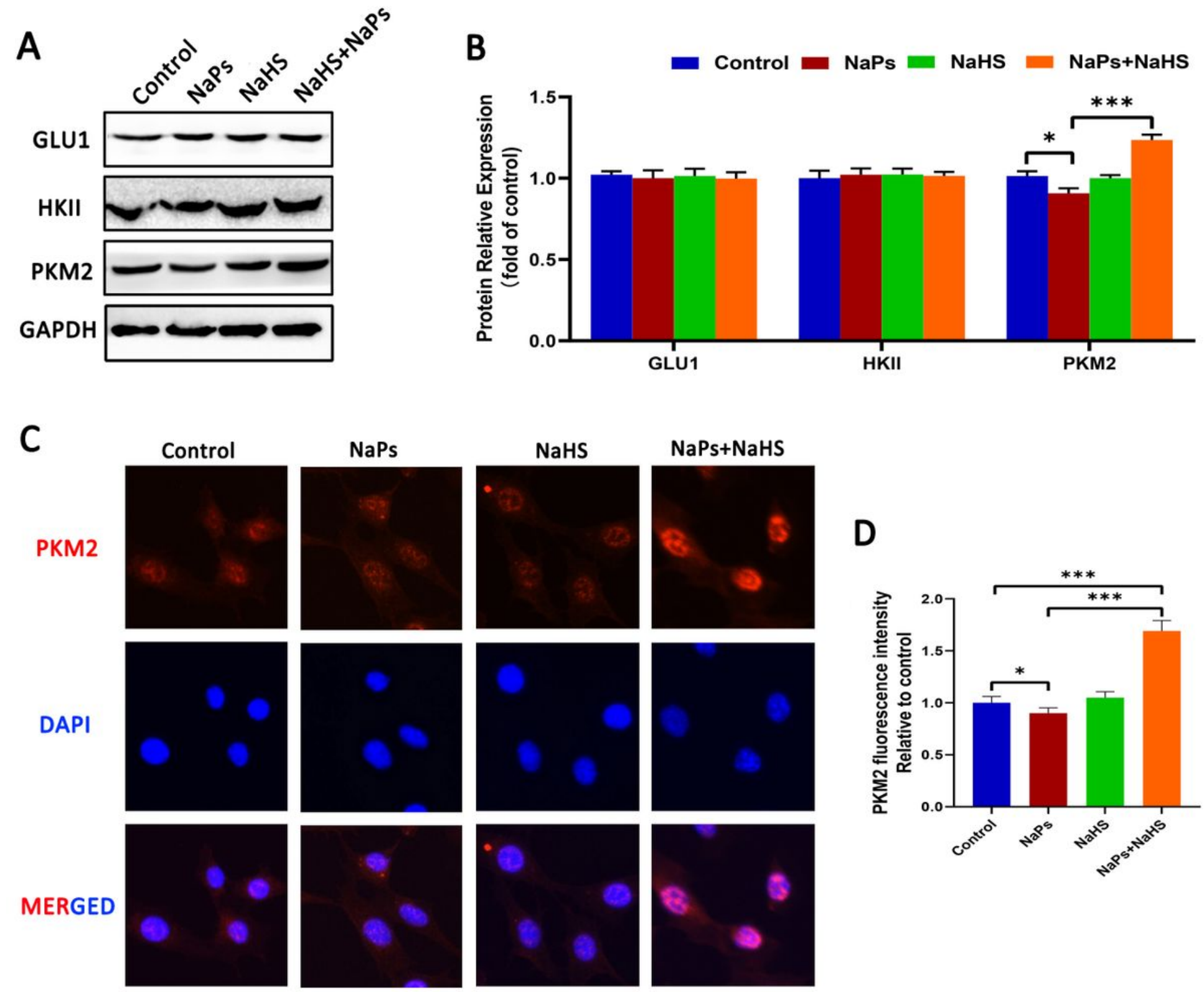

Figure 6

PKM2 signaling was involved in endogenous NaPs-triggered reproductive toxicity in vitro. (A) Nrf2 expression and location were examined by immunofluorescence analysis. (B) Statistical analysis of the fluorescence intensity of Nrf2 after treatment. (C) Western blotting was performed to determine GLU1, HKII and PKM2 proteins expressions. (D) The relative protein expressions were evaluated by Image J. $\triangle \mathrm{P}<0.05$, $\otimes \otimes \otimes P<0.001$. 

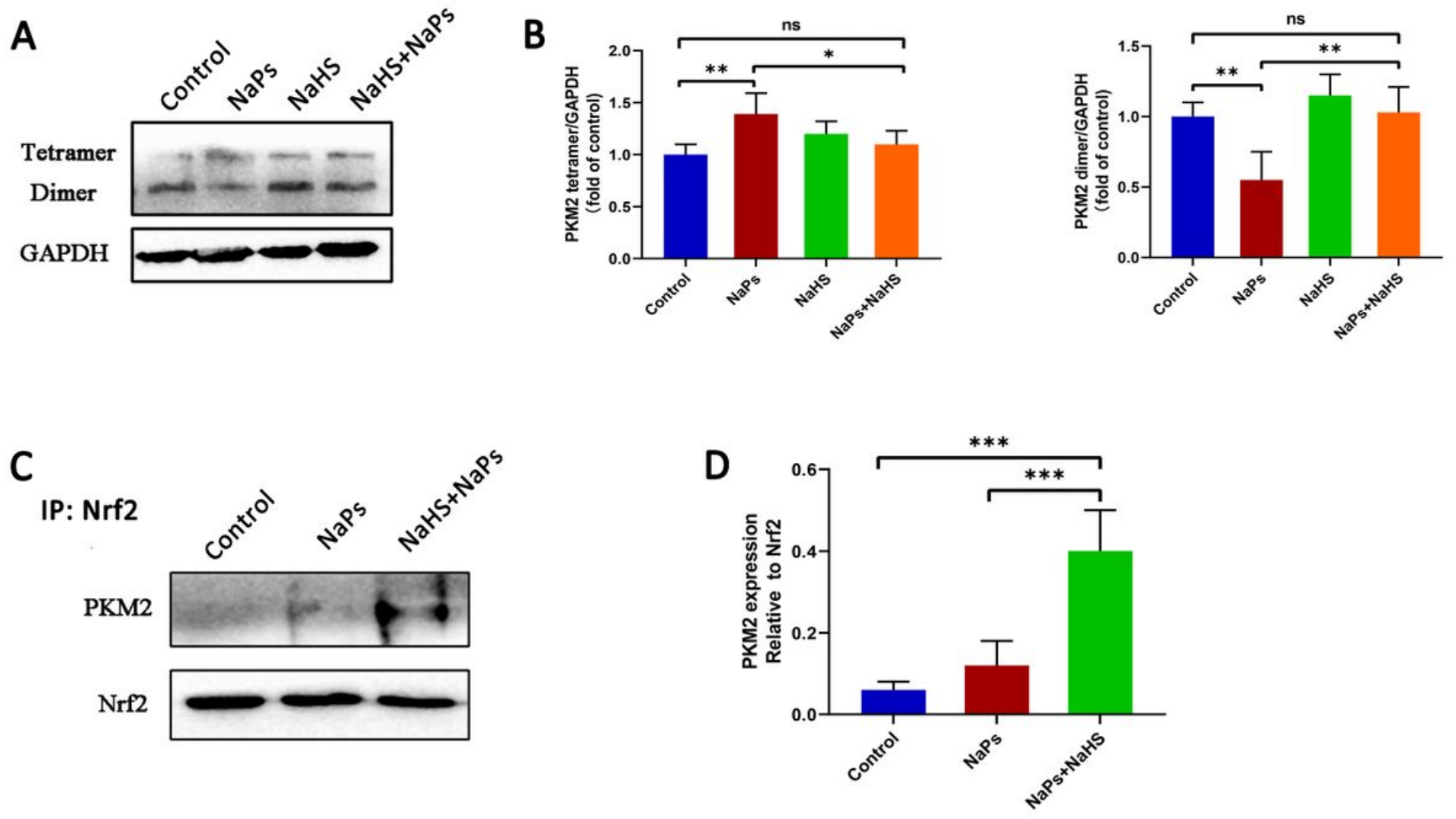

Figure 7

NaPs-altered dimerize and tetrameric PKM2 expressions were associated with Nrf2 signaling. (A) PKM2 dimer and tetramer formation were detected by Nondenature PAGE, and total PKM2 protein was analyzed by western blotting. (B) The relative protein expressions were evaluated by Image $\mathrm{J}$ software. (C) Co-IP analysis was performed to detect of the binding of Nrf2 with PKM2. (D) The relative protein expressions were evaluated by ImageJ software. ns, not significant; $\mathbb{X P}<0.05, \mathbb{Q} \mathbb{P}<0.01, \mathbb{\triangle} \mathbb{\otimes} \mathrm{P}<0.001$. 


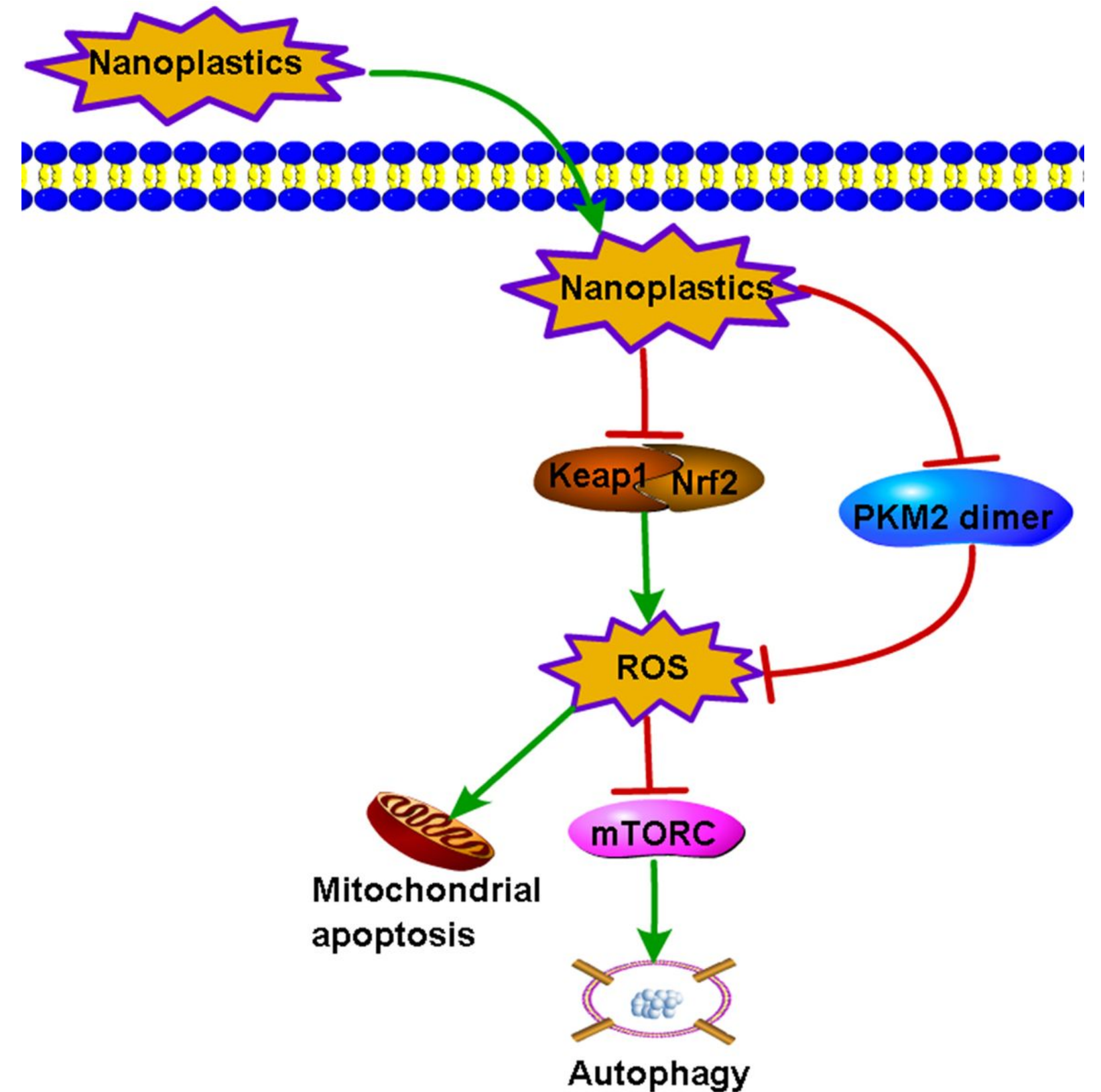

Figure 8

Schematic summary. The H2S donor NaHS drives the interaction of PKM2 and Nrf2 via promoting the dissociation of Nrf2 from Keap1, subsequently promotes the expression of $\mathrm{NqO} 1$ and $\mathrm{HO}-1$, and ameliorates the mitochondrial apoptosis and excessive autophagy induced by NaPs.

\section{Supplementary Files}

This is a list of supplementary files associated with this preprint. Click to download.

- SupplementalMaterials.doc 